\section{Akronyme in Klinischer Chemie und Laboratoriumsmedizin}

\author{
A. M. Gressner ${ }^{1}$ und O. A. Gressner Fevery ${ }^{2}$ \\ ${ }^{1}$ Labor Dr. Wisplinghoff Berlin, Berlin, Deutschland \\ ${ }^{2}$ Labor Dr. Wisplinghoff Köln, Köln, Deutschland
}

Synonym(e) Abkürzungen; Kurzbegriffe

\section{Englischer Begriff acronym}

Definition National und teilweise international gebräuchliche Kurzbegriffe/Abkürzungen in der Laboratoriumsmedizin.

\section{Beschreibung}

In Fachliteratur und Kommunikation werden auch im Bereich der Laboratoriumsmedizin/Klinischen Chemie eine Vielzahl von Akronymen verwendet, die sich auf folgende Beispiele beziehen: Analytische Methoden und Messgrößen (z. B.
AAS, EIA, EMIT, ELISA, ISE, MS), Kenngrößen (Hb, MCV, MCHC, AST, GOT, ALT, GPT, LDL, HDL), Reagenzlösungen (PBS, EDTA, TCA), Krankheitsbilder (AIDS, MS), Fachgesellschaften (AML, DGKL, VDGH, DGTI) u. v. a. Auch wenn die Nutzung von Akronymen die Kommunikation technisch und zeitlich sehr erleichtert, ist die Gefahr der Doppeldeutigkeit zu beachten, wenn die Kurzbegriffe aus einem Zusammenhang entfernt werden (z. B. MS = Massenspektrometrie vs. multiple Sklerose, $\mathrm{MCV}=$,mean corpuscular volume“"vs. mutiertes citrulliniertes Vimentin, AML = akute myeloische Leukämie vs. Arbeitsgemeinschaft medizinischer Laboratorien). Allerdings verhindern Verbindungen vieler Akronyme mit Dimensionsangaben, Einheiten, Messwerten eine fehlerhafte Zuordnung bzw. Fehlinterpretation eines Akronyms.

\section{Literatur}

Rolf $\mathrm{H}$ Lexikon medizinisch-wissenschaftlicher Akronyme, 4. Aufl. ISBN 3794518438, Schattauer Verlag 